\title{
Modeling and Simulation as Support for Development of Human Health Space Exploration Projects
}

\author{
Agostino G. Bruzzone Marina Massei \\ DIME, University of Genoa, Italy, \{agostino, massei\} @itim.unige.it \\ Giuseppina Mùrino Riccardo Di Matteo Matteo Agresta Giovanni Luca Maglione \\ Simulationteam, Italy, \\ \{giuseppina.murino; riccardo.dimatteo; matteo.agresta;giovanniluca.maglione\} \\ dsimulationteam.com
}

\begin{abstract}
The capability of the space environment to alter the cells behavior seems to be an opportunity for future researches in biology, for diseases such as cancer. This paper highlights the importance of Interoperable Simulation Systems as precious instruments to support and improve space exploration projects devoted to biological researches. The research investigates the potential of Modeling \& Simulation to reproduce a virtual environment to support Nano-satellite experiments in cooperation among the different stakeholders involved in a space mission, such as scientist, engineers and biologists.
\end{abstract}

Keywords: modeling \& simulation, HLA, space exploration; human health; cancer development and progression

\section{Introduction}

The sphere of the human environment and exploration continues to expand towards space; there is a need to enrich the knowledge on the effect of the Sun and "space weather" to preserve the safety of the astronauts (Marhavilas, 2004). The space environment conditions are extremely challenging for human body; indeed microgravity condition are joined with ionizing radiation sources including cosmic rays and solar particle events (SPEs) (Benton et al., 2001; Townsend, 2005). Several studies conducted on astronauts after they spent several months in space proved that an extended exposure to microgravity conditions are correlated with health problem, for example bone loss (Lloyd et al., 2008; Lang et al., 2004)

Despite the health of the astronauts, the space weather is a critical issue, its capability to alter the cells behaviour seems to be an opportunity for future researches in biology, for diseases such as cancer. This is proved by several studies, present in literature, describing experiments on microgravity devoted to gain insights into its effect on living organisms. (NASA, 2001).

Furthermore, several promising experiments have found some correlation with the behavior of certain cells and bacteria and the simultaneous conditions of microgravity joined with ionizing environment. The experimental results suggest that cell development and proliferation is different in microgravity conditions and within ionizing environment (Massimiani et al., 2014; Leys et al., 2009; Vanhavere et al., 2008; Mastroleo et al., 2009).

Some scientist supposes that microgravity conditions may give the possibility of developing

tissues and biological samples in three dimensions as it happens within human bodies: analyzing the experiments conducted in a cell cultures in a 1-g environment the proliferation is evolving only in two dimensions, this does not make them perfectly representative of what actually happens inside our body where the growth is tridimensional. The same cell types in orbital systems highlights this substantial difference confirming that space is a unique and incomparable environment for biological research.

The reasons of this different behavior have not yet been fully determined, but it is supposed to be correlated to a number of investigated factors:

- Interaction with terrestrial magnetic field: it could cause other effects in addition to those caused by microgravity (considering the nature of membranes which act as electrical capacity);

- Microgravity: this element generates different behaviors in biomedical samples between real and simulated microgravity; indeed in the ground simulators it is not possible to reach the microgravity levels common in low-Earth orbits (on the range of $109-105 \mathrm{~g}$ ). By altering gravity, we are able to investigate partially these effects on biological systems related to the presence and reaction to this unique force. However simulating microgravity on Earth for more than several seconds is impossible with existing technology. So by using spaceflights, we are starting to understand that not only gravity, but also the physical changes that occur in microgravity conditions, may have effects on the evolution of species and their ecologies 
- Joint effect of microgravity and ionizing environment: the impossibility to reproduce the effects of microgravity in a laboratory (due to technological limits) does not allow to consider its combined interactions with ionizing radiations. In addition, the space radiation is different from the one that we normally experience on Earth, such as $\mathrm{x}$-rays or $\gamma$-rays. The combined effect could result in additional elements affecting the radiation hazard caused by exposure; these are usually acting by "changing of body systems functioning at all levels: from cellular up to organism". In facts the ionizing radiation exposure causes changing on body systems.

All these factors, combined with the reduction of costs in space missions, increase the interest of biomedical research in using the space as a laboratory for its studies. Therefore the use of simulation could guarantee a right first time approach in setting up the experimentation \& testing.

\section{Nanosatellites for Biomedical Experimentation}

The world's first artificial satellite, the Sputnik 1, was put in space by Soviet Union in 1957. Since then, thousands of satellites have been launched into the space and are nowadays in orbit around the Earth (Lanius et al., 2013).

Last technological innovation, in use to support biomedical experimentations, is represented by CubeSat nanosatellite generation (Puig-Suari,et al., 2001). These miniaturized satellites for space research, usually launched by a carrier rocket or launch vehicle are made up of multiples of $10 \times 10 \times 11.35 \mathrm{~cm}$ cubic units (Bouwmeester and Guo, 2010). They have a mass of about 1 kilograms per unit and a Geocentric Orbit at Low Earth Orbit (LEO) altitude, usually $<0.1$ times Earth radius.

\section{Modelling \& Simulation Supporting Space Missions}

Space missions are extremely costly and dangerous and Modeling \& Simulation (M\&S) is extremely useful to support engineering and reduce risks; indeed $M \& S$ allows to evaluate feasibility of experimentation in terms of equipment and technical solutions (Baxter, 2010)

Decision makers as well as project leaders usually face limited resources and rigid time constraints for space experimentation; so they need to test the feasibility of complex systems before realizing them in order to avoid unexpected problems. That's why M\&S provides a strong support, particularly in the initial phase of a project giving to stakeholders a holistic view of the whole context (Montgomery, 2000)

In previous researches the authors performed several studies on $\mathrm{M} \& \mathrm{~S}$ reproducing complex systems behavior in different fields including space (Bruzzone et al., 2016), logistics (Bruzzone et al., 2014), Intelligent Agent Computer Generated Forces (IACGF), disaster recovery in critical environment (Bruzzone et al., 2016), reproduction of intelligent behavior (Wooldrige and Jennings, 1995; Bruzzone et al., 2015), data \& communications exchange among different entities (Bruzzone et al., 2013) and training (Bruzzone et al., 2011; Bruzzone et al., 2016). Despite all these areas are really different one each other, they have a common line because they reproduce complex problems where non-linear functions lead often to counter-intuitive behaviors on the system itself that evolves dynamically along the simulated timeline. Furthermore, the models allows to simulate conditions and situations that are often impossible to be reproduced in experiments on the Earth, both for the costs and for technological complexities.

To this end, the authors propose a simulation devoted to investigate all the operations required for setting the real experimentation of the Nano-satellite technology applied in space for studying the combined effect of microgravity and ionizing radiation on cancer cells affected by GBM (Glioblastoma Multiforme).

GBM is the most common form of malignant brain tumors with a median survival time of patients with less than one year. It represents $52 \%$ of all cases of primary brain tumor and $20 \%$ of all intracranial tumors. This type of cancer is actually treated by the best health facilities through the surgery and subsequent exposure to chemotherapeutic and radio therapies (Mahaley et al., 1989)

Because of its nature "multifaceted", complete surgical tumor excision is often very difficult and sometimes impossible without permanent damage in the patient. Because of the high incidence of this type of cancer and its characteristics it is necessary to deepen the knowledge of its pathogenesis by studying the behavior in various environments including the space. A better and detailed knowledge of cells behavior affected by GBM, should lead to identifying the causes that generate it or pharmacological remedies to counteract their evolution and development.

For this reason, the basic idea is referred to the biological effects of ionizing radiation and microgravity that could increase the chances of success of treatments and biomedical applications.

\section{Nanosatellites for Space Experimentation}

In this paragraph the overall architecture and nature of the CubeSat Systems are described. Indeed, it is 
proposed a summary of systems, subsystems and components system that have to be considered for being simulated:

- Mechanical Systems and Structure: the satellite is made mainly of Aluminum 6061T6; it is usually considered, also in these nanosatellites, by using rapid prototyping design to adopt space qualified materials to build some structural components and to reduce its weight. Biological experiments should be hosted in special insulated containment module that are designed to be equipped with monitoring system.

- Observation System: an onboard observation system is required to monitor cell samples at various stages of the experiment.

- Thermal and Heat Exchange Systems: to keep alive the cells during all the mission phases in necessary to maintain a controlled environment (around 37 ${ }^{\circ} \mathrm{C}$ ) despite the space extreme variation in terms of temperature. The thermal control system consists of sensors, insulating material, heat exchangers.

- Ionization and Radiations Phenomena: to obtain accurate scientific results, experimenters must understand the radiation environment during the duration of the experiment. A micro-dosimeter is necessary to measure the amount of ionizing radiation that is absorbed by the samples. The number and type of micro-dosimeters will depend on the required accuracy.

- Microgravity Environment: In order to understand microgravity conditions during the experiment, researchers need to use different kinds of accelerometers, with accuracy and dimensions compatible with mission goals. In particular two kinds of accelerometers are used. One for high level of acceleration during the launch, the other to measure microgravity conditions.

- Power Systems: an autonomous power system is needed to operate the whole systems including life support for keeping the cells alive during all mission phases. The nanosatellite uses solar cells and lithium batteries to provide power. The power is managed by a batteries charge regulator. During the integration and launch phases the nanosatellite is turned off and for this reason an umbilical connection with the launch site will provide power to the payload before the satellite release in orbit. Different solutions for umbilical connection need to be analyzed taking into account also the possible launch vehicle available.

- Telemetry, Tracking and Communications System (TT\&CS): the TT\&C act as the unique communication system once the $\mathrm{SC}$ is released from launch vehicle system. The TT\&C communication uplink allow the mission control center ground station to upload command sequences in order to program all the $\mathrm{SC}$ operations using a UHF/VHF radio link and also for the monitoring of the platform, Telemetry.

- On Board Computer (OBC): the On Board Computer act as the brain of the system coordinating the function of all the subsystems. The main features of the $\mathrm{OBC}$ include the presence of two independent, but cooperative cores: one low power consumption microcontroller for the general management of the satellite (payloads, TT\&C, specimen status, retrieving of data from sensors); the second core is an FPGA (Field Programmable Gate Array) for implementing specific tasks or generic systems also with IP cores (Intellectual Property) of third parties.

- The OBC is provided with several sensors on board and a 9 degree IMU (Inertial Measurement Unit) made by 3 axes magnetometer, 3 axes accelerometer and 3 axes gyroscope.

- Attitude and Orbit Determination and Control System (AODCS): this module supports the inorbit control of the satellite by using on-board sensors and actuators. This module support the orbit corrections, attitude and spin rate control with high accuracy to ensure the communications link and to properly analyze the results of environment monitoring system (e.g. radiation level). The AODCS controls the positioning of the satellite in the selected orbit, in order to understand the results from the micro-dosimeter according to the known radiation exposure levels.

- On Board Software for Power Systems (OBSW): this system is compatible with OBC hardware and is based on a firmware that could be updated in terms of functions along the mission if necessary.

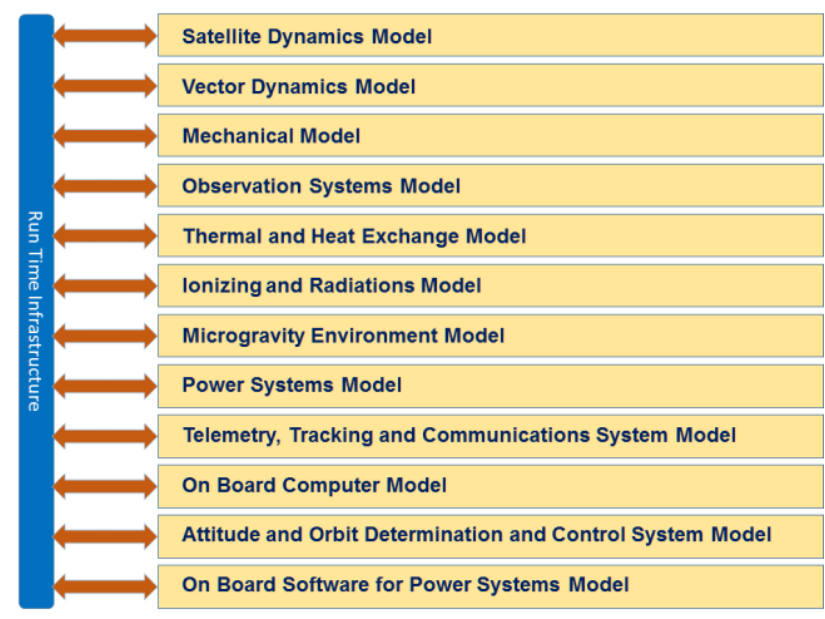

Figure 1. General Architecture. 


\section{General Architecture and Model Description}

M\&S supports strongly experimentation of space mission, giving the possibility to model the systems, subsystems and components and analyze interactions among them and biological samples.

The model is used to provide a measure of the resilience of the system to a hostile environment such as space, where reliability is challenging and it is necessary to evaluate redundancies, systems availability and capabilities.

Considering the flexibility demonstrated by the Intelligent Agents and Virtual Simulators developed within the MS2G (Modeling and Interoperable Simulation and Serious Games) paradigm (Bruzzone et al., 2014), the authors decide to realize a federation to be applied to Space Experimentation based on this approach. The authors benefits of their experience on past different simulators (Elfrey et al. 2011; Bruzzone et al., 2014; Bruzzone et al., 2016) created for SEE/Smackdown initiatives including SPIRALS (Space Interoperable Refilling and Advanced Logistics Simulator), IPHITOS (Interoperable simulation of a Protection solution based on light Interceptor Tackler operating in Outer Space) and SISMA (Medical Simulator of Astronaut including treatments, analysis and sickness models).

The simulator proposed in this case is designed to adopt the HLA IEEE1516 (High Level Architecture) standards and guarantees interoperability; the Federates could be based on stochastic models adopting combined simulation (continuous and discrete events combined together). The VV\&A (Verification, Validation and Accreditation) for this simulation will be conducted along the entire FEDEP (Federation Development Process) by using SME (Subject Matter Experts) from Simulation Team (Bruzzone et al., 2014)

\section{Description of the Different Models}

In this paragraph all the models are described. It is important to outline that for the purpose of this simulator the biological specimen encapsulated in the Nano-satellite are considered as a "black box" representing a reference for the onboard systems in terms of temperature to be maintained and data to be collected. The simulation models include:

- Satellite Dynamics Model: the model regulates the physics of the satellite including motion and acceleration based on all its characteristics.

- Vector Dynamics Model: a specific model for the vector is included to reproduce the release process.

- Mechanical Model: Mechanical Systems devoted to release the CubeSat from the vector, the umbilical connections for power support during launch and interactions with movable parts.
- Observation Systems Model: simulates the sensors that are interacting directly with the black box constituting the biological specimen as well as the links of the sensors to the CubeSat Core Systems devoted to conduct measurements during the experiments on the specimen. This model should include failures and performance estimations related to the experimentation on the cells based on the data collection, boundary conditions, status of components and sensors.

- Thermal and Heat Exchange Model: considers the thermal effects and heat exchange in the CubeSat with special attention to the cells for the experimentation. The aim of this module is to control the temperature of the cells at a constant value of $37^{\circ} \mathrm{C}$ balancing the radiated heat from and to the CubeSat.

- Ionizing and Radiations model: is devoted to evaluate performances of the sensors respect the "solar weather" and the estimated exposure to the radiation of the specimen. In addition these models could reproduce the effect of radiations in terms of noise over the signals.

- Microgravity Environment model: it is the model of the sensors adopted to measure microgravity acceleration.

- Power Systems model (PS): the model deals with the computation of power absorbed and consumed/provided by the battery. It considers the power request to keep the temperature of the cell constant as well as consumptions due to sensor operations and communications. It considers then dynamic charge-discharge curve of the lithium battery in the cell depending from solar power and conditioning system consumption; this model coupled with the satellite dynamic model allows to estimate the exposure of the solar panels to the sunshine and their efficiency in the different asset configuration respect Sun and Earth relative positions.

- Telemetry, Tracking and Communications System (TT\&CS) model: reproduces the datalink, a crucial component for correct operational profile. The cyber space is modeled to analyze the performances of the communication systems: it allows to visualize the information packet flow and evaluate communication system performances changing parameters characterizing the communication nodes and links in terms of availability, reliability and confidentiality. TT\&CS are coupled with the dynamic evolution of the CubeSat around the Earth and respect the Ground Base that receive the experimental data to identify when/how communicate; the coupling with the Power System Model allows to consider the relative power consumption. 
- On Board Computer model is crucial to implement and test on board computer system in a simulated environment before executing the mission. It also enables the possibility to evaluate the performance of Artificial Intelligence module (AI) devoted to direct dynamically the operation based on the level of decisional autonomy of the system during mission stochastic events.

- Attitude and Orbit Determination and Control System model (AODCS) simulates the performances of the sensors and modules devoted to control the orbit during the dynamic evolution of the mission. It could be used by reverse engineering to define the requirements of this system to achieve a specific overall performance.

- On Board Software for Power Systems model (OBSW) simulates the control firmware addicted to the control system for the Power System Module

Table 1.Description of the Simulation Parameters

\begin{tabular}{|c|c|}
\hline Solar Exposition & Dynamic Model \\
\hline Earth Sun distance $[\mathrm{m}]$ & Ground Station lat., long. \& altitude \\
\hline Earth diameter $[\mathrm{m}]$ & $\begin{array}{l}\text { CubeSat latitude, longitude \& } \\
\text { altitude }\end{array}$ \\
\hline Exposure Average angle [rad] & $\begin{array}{l}\text { CubeSat Asset: Pitch, Yaw. } \\
\text { Roll[rad] }\end{array}$ \\
\hline Starting Shadow Angle [rad] & CubeSat linear and angular speeds \\
\hline Ending Shadow Angle [rad] & CubeSat Status of Operations \\
\hline 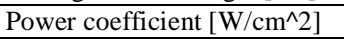 & Vector latitude, longitude \& altitude \\
\hline Solar Panel Surface $\left[\mathrm{cm}^{\wedge} 2\right]$ & Vector Asset: Pitch, Yaw. Roll[rad] \\
\hline number of panels & Vector linear and angular speeds \\
\hline \multicolumn{2}{|l|}{ Potential Solar Power [W] } \\
\hline $\begin{array}{l}\text { Current Efficiency of Solar } \\
\text { Panels }\end{array}$ & $\begin{array}{l}\text { Thermal Model and Heat } \\
\text { Exchange }\end{array}$ \\
\hline \multirow[t]{3}{*}{$\begin{array}{l}\text { Current Battery Charging } \\
{[\mathrm{mAh}]}\end{array}$} & Solar Constant [W m-2] \\
\hline & Stefan's coefficient $\left[\mathrm{W} \mathrm{m}^{\wedge}-2 \mathrm{~K}^{\wedge}-4\right]$ \\
\hline & T Sunshine Side of the CubeSat [K] \\
\hline SATCOM & T Shadow Side of the CubeSat [K] \\
\hline $\max$ distance $[\mathrm{m}]$ & Emissivity Factor \\
\hline max power consumption [W] & Radiation Factor \\
\hline Gain transmitter $[\mathrm{dBi}]$ & CubeSat Surface $\left[\mathrm{m}^{2}\right]$ \\
\hline Gain receiver $[\mathrm{dBi}]$ & Heat to be dispersed [W] \\
\hline frequency $[\mathrm{Hz}]$ & Insulation factor \\
\hline $\begin{array}{l}\text { N Exponent for Env. } \\
\text { Conditions }\end{array}$ & $\begin{array}{l}\text { Absorbed Energy on Exposed Face } \\
\text { [Wh] }\end{array}$ \\
\hline Power receiver [W] & $\begin{array}{l}\text { Dispersed Energy on shadow faces } \\
\text { [Wh] }\end{array}$ \\
\hline Communication Status & Current Energy Balance [Wh] \\
\hline Bandwidth $[\mathrm{Mb} / \mathrm{s}]$ & $\begin{array}{l}\text { Battery Use for Heat Exchange } \\
{[\mathrm{mAh}]}\end{array}$ \\
\hline \multicolumn{2}{|l|}{ Efficiency Level } \\
\hline Data to be Transmitted [Mb] & Battery \\
\hline Transmission Time [s] & Nominal Capacity [mAh] \\
\hline Power Consumption [W] & Operational Voltage [V] \\
\hline Energy Consumption [Wh] & Nominal Battery Energy [Wh] \\
\hline \multirow[t]{2}{*}{$\begin{array}{l}\text { Battery Use for SATCOM } \\
{[\mathrm{mAh}]}\end{array}$} & Initial State of Charge \\
\hline & Current capacity Level [mAh] \\
\hline Observation System Model & Current State of Charge \\
\hline \multicolumn{2}{|l|}{ Dbase Capacity [Mb] } \\
\hline Data to be Transmitted [Mb] & On Board Computer Model \\
\hline Current Data Flow [Mb/s] & $\begin{array}{l}\text { Availabilities of the different } \\
\text { Systems }\end{array}$ \\
\hline Observation System Status & Current Battery Use [mAh] \\
\hline
\end{tabular}

The models reproduce CubeSat dynamics in terms of orbit, power generation and consumption, battery recharging and communication management. It considers heating exchange and temperature control of the nanosatellite from the launching moment.

Major model parameters are summarized in Table 1. The simulation aims to evaluate the performance with special attention to the power required to keep the internal satellite temperature at $37^{\circ} \mathrm{C}$ and communicate with the ground station. The total power is obtained by the interaction of different systems models (e.g. AODCS, OBC, TT\&CS and PS). The energy to the different CubeSat systems is provided by battery and solar panels.

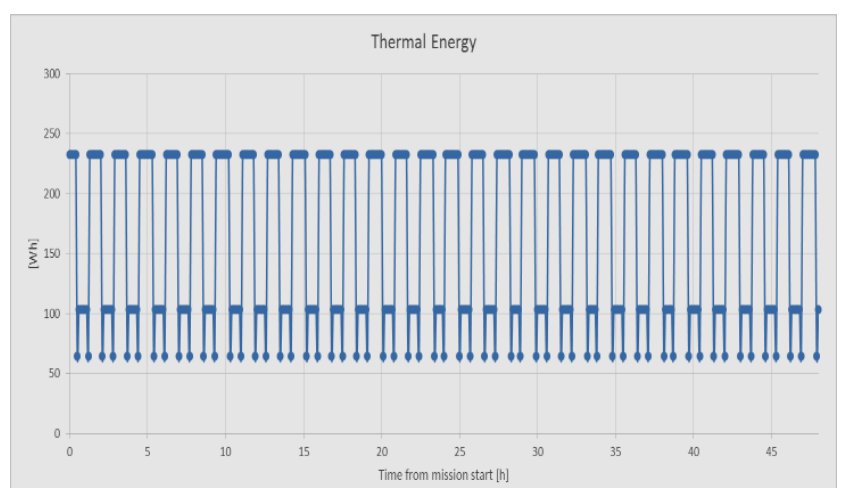

Figure 2. Generated Energy by Solar Panels

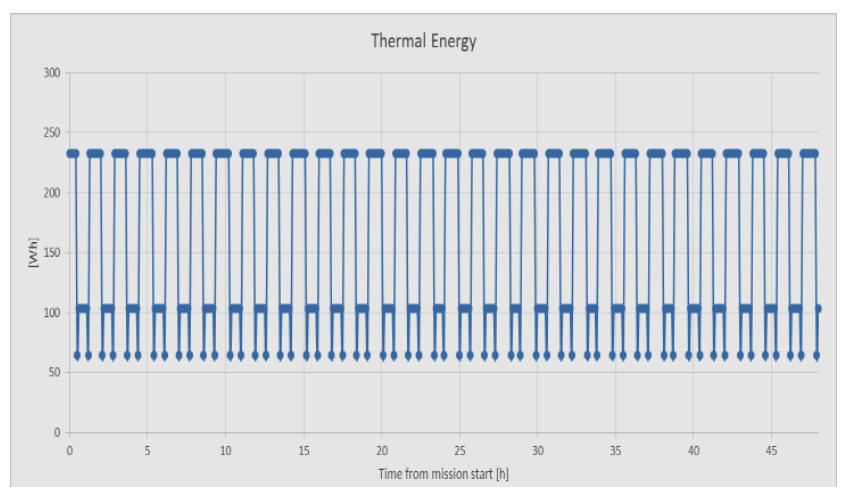

Figure 3.Thermal Energy Balance

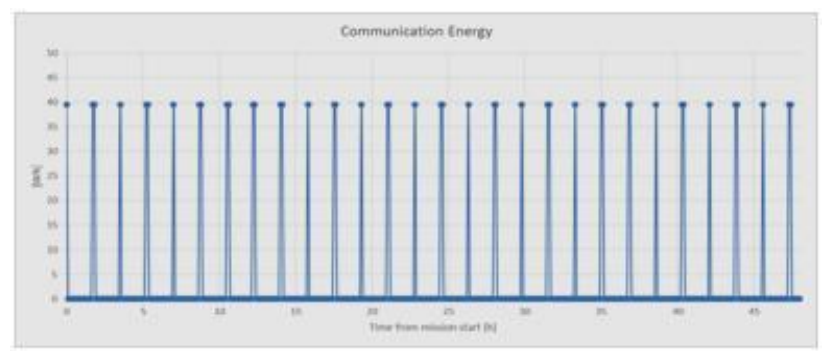

Figure 4. Energy required for Communications 


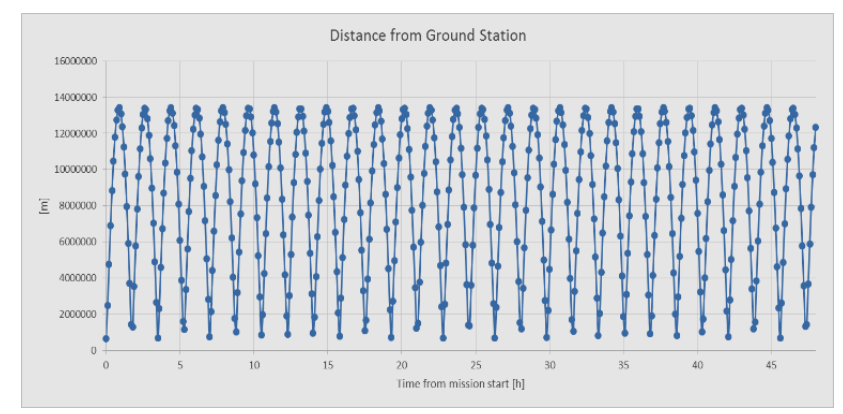

Figure 5. Communication Opportunities: CubeSatGround Base

The CubeSat is released by its vector when it is reached the proper operational orbit altitude; this process is activated by a simple spring mechanical device that releases it at a relative speed of $2.4 \mathrm{~m} / \mathrm{s}$ perpendicular to the vector trajectory.

Obviously the model computes the CubeSat orbit respect the movements of Earth and Sun, in order to evaluate dynamically the solar exposure and the conditions of low efficiency when the solar panels are affected by atmosphere (CubeSat relative sunset and sunrise); this allows to estimate the power generated by the solar panels in different conditions. In general there are different situations including darkness, (no charge), shadow (partial charge) and full exposure (full charge).

Under the hypothesis of omnidirectional antenna, the cyber layer is modelled to analyze the conditions when communications are possible as well as the exchange of information with the ground station considering the relative orbital position of the satellite.

Indeed, the TT\&CS model simulate all the data exchanges with the ground base, considering the different delay and the noise of the signal due to the position of the satellite. Since the CubeSat have a different rotation speed compared to the Earth, there are conditions where the satellite could not be able to transfer the data.

In these cases the TT\&CS close the communication with the Ground Base in order to save power and the OBC stores the experimental data in its Dbase for late transmission opportunities.

\section{Conclusions}

In the paper is described a simulation model of the systems devoted to conduct an experiment on tumor cells under micro-gravity and radiation conditions on a nanosatellite in space.

The conceptual model has been developed and implemented in order to support design, engineering, virtual prototyping and risk analysis about the different systems.

The simulation resulted very useful to create a virtual prototype to deal with the complexity of the different systems and their interactions as well as with the large number of the variables; in addition, the stochasticity related to the potential failures affects the mission success and requires proper risk analysis to identify most convenient satellite configurations and redundancies to mitigate problems.

By this approach it is possible to reduce risks and guarantee success in this context dealing with nanosatellite, so a pretty compact system of systems that needs to operate in space guaranteeing success despite limited budget. Indeed the use of simulation allows to improve design and reduce overall costs and risk; obviously it is fundamental to proper model the different systems and their interactions to proper reproduce the different operations and conditions even considering potential failures and interferences.

This paper proposes the preliminary models developed and their general structure; currently the authors are working on the interoperability of this simulator to construct a federation able to guarantee that the final result of this research will be flexible and open to be integrated with other interoperable simulators. Indeed by this approach the simulator will be modular and available to be adapted for reproducing other similar space experiments.

\section{References}

M. J. Baxter. Guidance for Space Program Modeling and Simulation. June 30, AEROSPACE REPORT NO. TOR2010(8591)-17, 2010.

E. R. Benton and E. V. Benton. Space radiation dosimetry in low-Earth orbit and beyond. Nuclear Instruments and Methods in Physics Research, section B, 184: 255-294, 2001.

J. Bouwmeester and J. Guo. Survey of worldwide pico-and nanosatellite missions, distributions and subsystem technology. Acta Astronautica, 67: 854-862, 2010.

A. Bruzzone. Intelligent Agents for Computer Generated Forces. Invited Speech at Gesi User Workshop, Wien, Italy, October 16-17, 2008.

A. Bruzzone, A. Tremori, and M. Massei. Adding Smart to the Mix. Modeling, Simulation \& Training: the International Defence Training Journal 3: 25-27, 2011.

A. Bruzzone, D. Merani, M. Massei, A. Tremori, C. Bartolucci C., and A. Ferrando. Modeling Cyber Warfare in Heterogeneous Networks for Protection of Infrastructures and Operations. In Proceedings of I3M'13 EMSS, Athens, September 25 - 27, 2013.

A. Bruzzone, F. Longo, M. Agresta, R. Di Matteo, and G.L. Maglione. Autonomous Systems for Operations in Critical Environments. In Proceedings of the Modeling and Simulation of Complexity in Intelligent, Adaptive and Autonomous Systems (MSCIAAS 2016) and Space Simulation for Planetary Space Exploration (SPACE 2016) Pasadena, California, April 03 - 06, 2016.

A. Bruzzone, F. Longo, M. Massei, L. Nicoletti, and M. Agresta. Safety and security in fresh good supply chain. International Journal of Food Engineering, 10: 545-556, 2014. 
A. Bruzzone, L. Dato, and A. Ferrando. Simulation Exploration Experience: Providing Effective Surveillance and Defense for a Moon Base against Threats from Outer Space. In Proceedings of IEEE/ACM 18th International Symposium on Distributed Simulation and Real Time Applications 01 - 03 October 2014, France, Toulouse.

A. Bruzzone, M. Massei , M. Agresta, A. Tremori, F. Longo, G. Murino, F. De Felice, and A. Petrillo. Human behavior simulation for smart decision making in emergency prevention and mitigation within urban and industrial environments. In Proc. of 27th EMSS European Modeling \& Simulation Simposium, 2015.

A. Bruzzone, M. Massei, A. Tremori, F.Longo, L. Nicoletti, S. Poggi, C. Bartolucci, E. Picco, and G. Poggio. MS2G: simulation as a service for data mining and crowd sourcing invulnerability Reduction. In Proceedings of WAMS2014, Istanbul, Turkey, September 16-19, 2014.

P. R. Elfrey, G. Zacharewicz, and M. Ni. Smackdown. Adventures in Simulation Standards. In S. Jain, R.R. Creasey, J. Himmelspach, K.P.White, and M. Fu (Eds.), Proceedings of the 2011 Winter Simulation Conference, 2011.

T. Lang, A. LeBlanc, H. Evans, Y. Lu, H. Genant, and A. $\mathrm{Yu}$. Cortical and trabecular bone mineral loss from the spine and hip in long-duration spaceflight. Journal of Bone and Mineral Research, 19:1006-1012, 2004.

R. D. Lanius, J. M. Logsdon, and R. W. Smith (Eds.), Reconsidering Sputnik: Forty years since the Soviet satellite. Routledge. 2013.

N. Leys, S. Baatout, C. Rosier, A. Dams, C. s'Heeren, R. Wattiez, and M. Mergeay. The response of Cupriavidus metallidurans $\mathrm{CH} 34$ to spaceflight in the international space station. Antonie van Leeuwenhoek International Journal of General and Molecular Microbiology, 96: $227-$ 245, 2009. DOI: 10.1007/s10482-009-9360-5. PMID: 19572210.

S. A. Lloyd, E. R. Bandstra, N. D. Travis, G. A. Nelson, J. D. Bourland, M. J Pecaut, and T. A. Bateman. Spaceflightrelevant types of ionizing radiation and cortical bone: Potential LET effect?. Advances in Space Research, 42: 1889-1897, 2008.

M. S. Mahaley Jr, C. Mettlin, N. Natarajan, E. R. Laws Jr, and B.B. Peace. National survey of patterns of care for brain-tumor patients. Journal of neurosurgery, 71: 826836, 1989

P. K. Marhavilas. The Space Environment and its impact on human activity. 2004.

C. Massimiani, S. Gemini Piperni, M. Carnio, W. Zambuzzi, C. Cappelletti, and F. Graziani. Space systems design for research on the interaction of osteoblast-like cells and biomaterials (hydroxyapatite particles and titanium) in microgravity environment. IAC-14-A1-3-6, In Proceedings of the 64th International Astronautical Conference Toronto, September 2014.

F. Mastroleo, R. Van Houdt, B. Leroy, M. Benotmane, A. Janssen, M. Mergeay, F. Vanhavere, L. Hendrickx, R. Wattiez, and N. Leys. Experimental design and environmental parameters affect Rhodospirillum rubrum $\mathrm{S} 1 \mathrm{H}$ response to space flight. International Society for Microbial Ecology, 3: 1402-1419, 2009. DOI: 10.1038/ismej.2009.74.
D. C. Montgomery. Design and Analysis of Experiments. John Wiley \& Sons, New York (NY) 2000.

J. Puig-Suari, C. Turner, and W. Ahlgren. Development of the standard CubeSat deployer and a CubeSat class PicoSatellite. In Proc. of IEEE Aerospace Conference, Vol. 1, pp. 1-347, 2001.

J. M. Quero, L. León, J. Jiménez, M. Brey, J.M. Moreno, S. de la Rosa, A. Sánchez, D. López, and C. Leyva. CEPHEUS, a Multi-project Satellite for technology qualification. Acta Astronautica, 117: 238-242, 2015.

L. W. Townsend. Implications of the space radiation environment for human exploration in deep space. Radiation Protection Dosimetry, 115: 44-50, 2005.

F. Vanhavere, J. L. Genicot, D. O'Sullivan, D. Zhou, F. Spurny, I. Jadrnickova, G. Sawakuchi, and E. G. Yukihara. DOsimetry of BIological EXperiments in SPace (DOBIES) with luminescence (OSL and TL) and track etch detectors. Radiation Measurements, 43: 694-697, 2008. DOI: 10.1016/j.radmeas.2007.12.002.

M. Wooldrige and N. R. Jennings. Intelligent Agents: Theory and Practice. 1995.

The Drawing of a New Era of Research, Research Results Accomplishments: An Analysis of Results from 2000-2001, NASA technical records Practice. 